\title{
Corrigendum: Genome-wide association study identifies new HLA class II haplotypes strongly protective against narcolepsy
}

Hyun Hor, Zoltán Kutalik, Yves Dauvilliers, Armand Valsesia, Gert J Lammers, Claire E H M Donjacour, Alex Iranzo, Joan Santamaria, Rosa Peraita Adrados, José L Vicario, Sebastiaan Overeem, Isabelle Arnulf, Ioannis Theodorou, Poul Jennum, Stine Knudsen, Claudio Bassetti, Johannes Mathis, Michel Lecendreux, Geert Mayer, Peter Geisler, Antonio Benetó, Brice Petit, Corinne Pfister, Julie Vienne Bürki, Gérard Didelot, Michel Billiard, Guadalupe Ercilla, Willem Verduijn, Frans H J Claas, Peter Vollenwider, Gerard Waeber, Dawn M Waterworth, Vincent Mooser, Raphaël Heinzer, Jacques S Beckmann, Sven Bergmann \& Mehdi Tafti Nat. Genet. 42, 786-789 (2010); published online 15 August 2010; corrected after print 27 October 2010

In the version of this article initially published, the name of author Peter Vollenweider was incorrectly written as Peter Vollenwider. Also, Claudio Bassetti's affiliation was incorrectly listed as Neurocentro (Ente ospedaliero cantonale) della Svizzera Italiana, Ospedale Civico, Lugano, Switzerland. His correct affiliation is Department of Neurology, University Hospital Zurich, Zurich, Switzerland. These errors have been corrected in the HTML and PDF versions of the article.

\section{Corrigendum: Twelve type 2 diabetes susceptibility loci identified through large-scale association analysis}

Benjamin F Voight, Laura J Scott, Valgerdur Steinthorsdottir, Andrew P Morris, Christian Dina, Ryan P Welch, Eleftheria Zeggini, Cornelia Huth, Yurii S Aulchenko, Gudmar Thorleifsson, Laura J McCulloch, Teresa Ferreira, Harald Grallert, Najaf Amin,Guanming Wu, Cristen J Willer, Soumya Raychaudhuri, Steve A McCarroll, Claudia Langenberg, Oliver M Hofmann, Josée Dupuis, Lu Qi, Ayellet V Segrè, Mandy van Hoek, Pau Navarro, Kristin Ardlie, Beverley Balkau, Rafn Benediktsson, Amanda J Bennett, Roza Blagieva, Eric Boerwinkle, Lori L Bonnycastle, Kristina Bengtsson Boström, Bert Bravenboer, Suzannah Bumpstead, Noisël P Burtt, Guillaume Charpentier, Peter S Chines, Marilyn Cornelis, David J Couper, Gabe Crawford, Alex S F Doney, Katherine S Elliott, Amanda L Elliott, Michael R Erdos, Caroline S Fox, Christopher S Franklin, Martha Ganser, Christian Gieger, Niels Grarup, Todd Green, Simon Griffin, Christopher J Groves, Candace Guiducci, Samy Hadjadj, Neelam Hassanali, Christian Herder, Bo Isomaa, Anne U Jackson, Paul R V Johnson, Torben Jørgensen, Wen H L Kao, Norman Klopp, Augustine Kong, Peter Kraft, Johanna Kuusisto, Torsten Lauritzen, Man Li, Aloysius Lieverse, Cecilia M Lindgren, Valeriya Lyssenko, Michel Marre, Thomas Meitinger, Kristian Midthjell, Mario A Morken, Narisu Narisu, Peter Nilsson, Katharine R Owen, Felicity Payne, John R B Perry, Ann-Kristin Petersen, Carl Platou, Christine Proença, Inga Prokopenko, Wolfgang Rathmann, N William Rayner, Neil R Robertson, Ghislain Rocheleau, Michael Roden, Michael J Sampson, Richa Saxena, Beverley M Shields, Peter Shrader, Gunnar Sigurdsson, Thomas Sparsø, Klaus Strassburger, Heather M Stringham, Qi Sun, Amy J Swift, Barbara Thorand, Jean Tichet, Tiinamaija Tuomi, Rob M van Dam, Timon W van Haeften, Thijs van Herpt, Jana V van Vliet-Ostaptchouk, G Bragi Walters, Michael N Weedon, Cisca Wijmenga, Jacqueline Witteman, The MAGIC investigators, The GIANT Consortium, Richard N Bergman, Stephane Cauchi, Francis S Collins, Anna L Gloyn, Ulf Gyllensten, Torben Hansen, Winston A Hide, Graham A Hitman, Albert Hofman, David J Hunter, Kristian Hveem, Markku Laakso, Karen L Mohlke, Andrew D Morris, Colin N A Palmer, Peter P Pramstaller, Igor Rudan, Eric Sijbrands, Lincoln D Stein, Jaakko Tuomilehto, Andre Uitterlinden, Mark Walker, Nicholas J Wareham, Richard M Watanabe, Gonçalo R Abecasis, Bernhard O Boehm, Harry Campbell, Mark J Daly, Andrew T Hattersley, Frank B Hu, James B Meigs, James S Pankow, Oluf Pedersen, H-Erich Wichmann, Inês Barroso, Jose C Florez, Timothy M Frayling, Leif Groop, Rob Sladek, Unnur Thorsteinsdottir, James F Wilson, Thomas Illig, Philippe Froguel, Cornelia M van Duijn, Kari Stefansson, David Altshuler, Michael Boehnke \& Mark I McCarthy Nat. Genet. 42, 579-589 (2010); published online 27 June 2010; corrected after print 27 August 2010

In the version of this article initially published, there was an error in Table 1. Specifically, for rs5945326, the risk and non-risk alleles were reversed. The correct risk allele at rs5945326 is A, the non-risk allele is G and the risk allele frequency in HapMap CEU is 0.79 . These errors have been corrected in the HTML and PDF versions of the article.

Erratum: Genome-wide association identifies multiple ulcerative colitis susceptibility loci

Dermot P B McGovern, Agnès Gardet, Leif Törkvist, Philippe Goyette, Jonah Essers, Kent D Taylor, Benjamin M Neale, Rick T H Ong, Caroline Lagacé, Chun Li, Todd Green, Christine R Stevens, Claudine Beauchamp, Phillip R Fleshner, Marie Carlson, Mauro D’Amato, Jonas Halfvarson, Martin L Hibberd, Mikael Lördal, Leonid Padyukov, Angelo Andriulli, Elisabetta Colombo, Anna Latiano, Orazio Palmieri, Edmond-Jean Bernard, Colette Deslandres, Daan W Hommes, Dirk J de Jong, Pieter C Stokkers, Rinse K Weersma, The NIDDK IBD Genetics Consortium, Yashoda Sharma, Mark S Silverberg, Judy H Cho, Jing Wu, Kathryn Roeder, Steven R Brant, L Phillip Schumm, Richard H Duerr, Marla C Dubinsky, Nicole L Glazer, Talin Haritunians, Andy Ippoliti, Gil Y Melmed, David S Siscovick, Eric A Vasiliauskas, Stephan R Targan, Vito Annese, Cisca Wijmenga, Sven Pettersson, Jerome I Rotter, Ramnik J Xavier, Mark J Daly, John D Rioux \& Mark Seielstad Nat. Genet. 42, 332-337 (2010); published online 14 March 2010; corrected after print 29 March 2011

In the version of this article initially published, Kathryn Roeder's affiliation was incorrect. Her correct affiliation is the Department of Statistics, Carnegie Mellon University, Pittsburgh, Pennsylvania, USA. The error has been corrected in the HTML and PDF versions of the article. 